\title{
On the Ramsey Number $R(4,6)$
}

\author{
Geoffrey Exoo \\ Department of Mathematics and Computer Science \\ Indiana State University \\ Terre Haute, IN 47809 \\ ge@cs.indstate.edu
}

Submitted: Mar 6, 2012; Accepted: Mar 23, 2012; Published: Mar 31, 2012

Mathematics Subject Classifications: 05C55, 05D10

\begin{abstract}
The lower bound for the classical Ramsey number $R(4,6)$ is improved from 35 to 36 . The author has found 37 new edge colorings of $K_{35}$ that have no complete graphs of order 4 in the first color, and no complete graphs of order 6 in the second color. The most symmetric of the colorings has an automorphism group of order 4 , with one fixed point, and is presented in detail. The colorings were found using a heuristic search procedure.
\end{abstract}

This note deals with a new lower bound for the classical Ramsey number $R(4,6)$. Recall that the classical Ramsey number $R(s, t)$ is the smallest integer $n$ such that in any two-coloring of the edges of $K_{n}$ there is a monochromatic copy of $K_{s}$ in the first color or a monochromatic copy of $K_{t}$ in the second color. A recent summary of the state of the art for Ramsey numbers can be found in the Dynamic Survey [10].

Theorem 1. $R(4,6) \geq 36$.

Proof. The proof is given by the coloring of $K_{35}$ which can be derived from Table 1, below. This table contains an adjacency list for the color one graph of a two-coloring of $K_{35}$. All other edges are assigned color two.

This coloring improves the lower bound for $R(4,6)$ from 35 to 36 , five short of the upper bound of 41 [8]. The coloring has an automorphism group of order 4 , with exactly one fixed point [7]. It was found using a method that the author had discarded many years ago, a method we now discuss.

A number of the lower bounds given in the table of two color classical Ramsey numbers (see Table 1 in the Dynamic Survey [10]) were established by this author using computer search techniques. Three of these, $R(4,6), R(3,10)$, and $R(5,5)[1,2,3]$, are the smallest unsettled cases for two color classical Ramsey numbers. They are also, in the opinion of this author, the only unsettled cases where optimal colorings can be found using computer 


\begin{tabular}{|c|c|c|c|c|c|c|c|c|c|c|c|c|c|c|c|}
\hline $0:$ & 2 & 6 & 7 & 9 & 11 & 13 & 15 & 17 & 18 & 20 & 21 & 23 & 24 & 26 & 283 \\
\hline 1: & 3 & 4 & 5 & 9 & 11 & 13 & 15 & 17 & 18 & 21 & 22 & 23 & 25 & 27 & 2933 \\
\hline 2: & 0 & 4 & 5 & 8 & 10 & 12 & 14 & 16 & 19 & 20 & 21 & 22 & 25 & 27 & 2832 \\
\hline 3: & 1 & 6 & 7 & 8 & 10 & 12 & 14 & 16 & 19 & 20 & 22 & 23 & 24 & 26 & 2933 \\
\hline 4: & 1 & 2 & 7 & 8 & 10 & 11 & 13 & 17 & 19 & 20 & 22 & 24 & 26 & 31 & 34 \\
\hline 5: & 1 & 2 & 6 & 8 & 9 & 11 & 14 & 17 & 19 & 21 & 23 & 24 & 26 & 30 & 34 \\
\hline 6: & 0 & 3 & 5 & 9 & 10 & 11 & 12 & 16 & 18 & 21 & 23 & 25 & 27 & 31 & 34 \\
\hline 7: & 0 & 3 & 4 & 8 & 9 & 10 & 15 & 16 & 18 & 20 & 22 & 25 & 27 & 30 & 34 \\
\hline 8: & 2 & 3 & 4 & 5 & 7 & 9 & 12 & 15 & 17 & 23 & 24 & 27 & 29 & 31 & 32 \\
\hline 9: & 0 & 1 & 5 & 6 & 7 & 8 & 13 & 14 & 16 & 22 & 25 & 26 & 29 & 31 & 32 \\
\hline 10: & 2 & 3 & 4 & 6 & 7 & 11 & 13 & 14 & 18 & 21 & 25 & 26 & 28 & 30 & 33 \\
\hline 11: & 0 & 1 & 4 & 5 & 6 & 10 & 12 & 15 & 19 & 20 & 24 & 27 & 28 & 30 & 33 \\
\hline 12: & 2 & 3 & 6 & 8 & 11 & 13 & 15 & 17 & 18 & 20 & 22 & 25 & 31 & 33 & \\
\hline 13: & 0 & 1 & 4 & 9 & 10 & 12 & 14 & 16 & 19 & 21 & 23 & 24 & 31 & 33 & \\
\hline 14: & 2 & 3 & 5 & 9 & 10 & 13 & 15 & 17 & 18 & 20 & 22 & 24 & 30 & 32 & \\
\hline 15: & 0 & 1 & 7 & 8 & 11 & 12 & 14 & 16 & 19 & 21 & 23 & 25 & 30 & 32 & \\
\hline 16: & 2 & 3 & 6 & 7 & 9 & 13 & 15 & 19 & 21 & 24 & 27 & 29 & 32 & 34 & \\
\hline 17: & 0 & 1 & 4 & 5 & 8 & 12 & 14 & 18 & 20 & 25 & 26 & 29 & 32 & 34 & \\
\hline 18: & 0 & 1 & 6 & 7 & 10 & 12 & 14 & 17 & 22 & 24 & 27 & 28 & 33 & 34 & \\
\hline 19: & 2 & 3 & 4 & 5 & 11 & 13 & 15 & 16 & 23 & 25 & 26 & 28 & 33 & 34 & \\
\hline 20: & 0 & 2 & 3 & 4 & 7 & 11 & 12 & 14 & 17 & 23 & 27 & 29 & 30 & 31 & \\
\hline 21: & 0 & 1 & 2 & 5 & 6 & 10 & 13 & 15 & 16 & 22 & 26 & 29 & 30 & 31 & \\
\hline 22: & 1 & 2 & 3 & 4 & 7 & 9 & 12 & 14 & 18 & 21 & 26 & 28 & 30 & 31 & \\
\hline 23: & 0 & 1 & 3 & 5 & 6 & 8 & 13 & 15 & 19 & 20 & 27 & 28 & 30 & 31 & \\
\hline 24: & 0 & 3 & 4 & 5 & 8 & 11 & 13 & 14 & 16 & 18 & 26 & 27 & 32 & 33 & 34 \\
\hline 25: & 1 & 2 & 6 & 7 & 9 & 10 & 12 & 15 & 17 & 19 & 26 & 27 & 32 & 33 & 34 \\
\hline 26: & 0 & 3 & 4 & 5 & 9 & 10 & 17 & 19 & 21 & 22 & 24 & 25 & 28 & 29 & \\
\hline 27: & 1 & 2 & 6 & 7 & 8 & 11 & 16 & 18 & 20 & 23 & 24 & 25 & 28 & 29 & \\
\hline 28: & 0 & 2 & 10 & 11 & 18 & 19 & 22 & 23 & 26 & 27 & 29 & 31 & 34 & & \\
\hline 29: & 1 & 3 & 8 & 9 & 16 & 17 & 20 & 21 & 26 & 27 & 28 & 30 & 34 & & \\
\hline 30: & 5 & 7 & 10 & 11 & 14 & 15 & 20 & 21 & 22 & 23 & 29 & 34 & & & \\
\hline 31: & 4 & 6 & 8 & 9 & 12 & 13 & 20 & 21 & 22 & 23 & 28 & 34 & & & \\
\hline 32: & 0 & 2 & 8 & 9 & 14 & 15 & 16 & 17 & 24 & 25 & 33 & & & & \\
\hline 33: & 1 & 3 & 10 & 11 & 12 & 13 & 18 & 19 & 24 & 25 & 32 & & & & \\
\hline 34: & 4 & 5 & 6 & 7 & 16 & 17 & 18 & 19 & 24 & 25 & 28 & 29 & 30 & 31 & \\
\hline
\end{tabular}

Table 1: A $(4,6)$-coloring of $K_{35}$. 
methods that manipilate colorings one edge at a time. These three lower bounds were established using one (or more) of the following computer search methods. In each case, the object of the method is to produce colorings with no monochromatic subgraphs of order $s$ in color one, and no monochomatic subgraphs of order $t$ in color two. Monochromatic copies of such subgraphs in a coloring will be referred to as bad subgraphs.

Method A: One begins with a randomly generated edge coloring for a complete graph whose order $n$ is small enough so that a good coloring can be obtained easily. Then using simulated annealing [6], or a synthesis of simulated annealing and tabu search [5], the coloring is transformed into a good coloring by looking at individual edges, and choosing the color that minimizes the number of bad subgraphs. When all bad subgraphs have been eliminated, $n$ is incremented, and the process is repeated.

Method B: This method is different from Method A in two respects. Instead of beginning with a graph of small order, one begins with a complete graph of the desired order (e.g., large enough to improve the lower bound for the Ramsey number). But the important difference pertains to the objective function. Instead of simply recoloring edges so as to minimize the number of bad subgraphs, we add a term to the objective that attempts to maximize the number of monochromatic induced copies of $P_{4}$, the path on 4 vertices. The importance of the $P_{4}$ count in the objective can be as great or greater than the bad subgraph counts.

Method C: Here one begins by searching for highly symmetric colorings, for example circle colorings (or more generally, Cayley colorings), that have relatively few bad subgraphs, and that have one additional property. They must have individual edges (as opposed to orbits) that, when recolored, reduce the number of monochromatic subgraphs. Once such a coloring is found, one proceeds as in Method A.

One further remark on Method B should be made. Often we used a somewhat more detailed variation of the method. There are 11 isomorphism classes of graphs of order 4, and hence 11 essentially different ways to two-color the edges of a subgraph of order 4 in a two-coloring of $K_{n}$. If we count the number of vertex sets of size four which induce each of these 11 possible colorings, we produce a vector of length 11. Edges can be thus recolored so as to minimize the distance between the computed vector and a postulated target vector. The target vector might be determined by looking at known good colorings, by a higher level optimization process, or by sheer speculation. Experience has shown that maximizing the number of induced $P_{4}$ 's is the key ingredient.

All three methods have successfully produced lower bounds for classical Ramsey numbers. The current lower bound for $R(5,5)$, for example, was originally established [1] using Method C. Very soon thereafter, we were able to find the same coloring using Method B, and eventually, using Method A. Since the idea behind Method B does not seem to have any direct theoretical justification, and since Method A was faster (counting induced $P_{4}$ 's takes more time than counting cliques, when the number of cliques is small), Method B was gradually discarded. 


\begin{tabular}{|l|rrrrr|}
\hline 4-subgraph & $G_{1}$ & $G_{2}$ & $G_{3}$ & $G_{4}$ & $G_{5}$ \\
\hline$E_{4}$ & 0 & 0 & 0 & 0 & 0 \\
$K_{2}$ & 1886 & 1464 & 1475 & 1484 & 1500 \\
$2 K_{2}$ & 1640 & 1311 & 1303 & 1427 & 1273 \\
$P_{3}$ & 4880 & 4204 & 4228 & 4364 & 4208 \\
$K_{1,3}$ & 2167 & 2199 & 2215 & 2092 & 2247 \\
$P_{4}$ & 9553 & 9190 & 9138 & 9359 & 9152 \\
$K_{3}$ & 3204 & 2856 & 2834 & 3027 & 2804 \\
$K_{3}+e$ & 11268 & 11776 & 11784 & 11589 & 11770 \\
$C_{4}$ & 2558 & 2900 & 2920 & 2687 & 2936 \\
$K_{4}-e$ & 7634 & 8645 & 8644 & 8534 & 8667 \\
$K_{4}$ & 1586 & 1831 & 1835 & 1813 & 1819 \\
\hline
\end{tabular}

Table 2: Induced subgraph counts in five $(4,6)$-colorings of $K_{34}$.

However, Method B has at least one potential advantage. By manipulating the objective function, one can drive the search away from known colorings, and (hopefully) toward new colorings. So, given the significant increase in computer speed since the mid-1980's, the author decided to revisit Method B, and apply it to each of the three frontier cases mentioned above. In addition to attempting to improve the lower bounds, we were also interested in finding new examples of colorings that equaled the current bounds, hoping to find colorings that were in some respect different from the known examples. It was such a coloring that led to the improved lower bound for $R(4,6)$.

In Table 2, we present some data pertaining to the color two subgraphs from five new $(4,6)$-colorings of $K_{34}$ that were obtained together (on different machines, but at roughly the same time). This table lists the counts for each of the 11 types of induced subgraphs of order 4. The important column is that of $G_{1}$, the other four colorings are presented as representative examples. The colorings were obtained using a variety of target vectors (for counts of induced colorings of order 4), created more or less ad hoc. We emphasize that in the table, the graph described is the color two graph. The abbreviation $E_{4}$ denotes the empty (edgeless) graph of order 4 , and $P_{4}$ denotes the path of order 4 ; the other abbreviations are fairly standard and of lesser importance.

Observe that the induced subgraph counts are approximately the same in each case, except for $G_{1}$. The other four have subgraph counts that are substantially the same as the hundreds of other $(4,6)$-colorings the author found previously. The coloring $G_{1}$ is quite different from any of these. The $K_{4}$ count is only 1586 , whereas in no other case did we find a coloring with less than 1780 monochromatic $K_{4}$ 's (in the $K_{6}$-free color). In addition, the number of induced $P_{4}$ 's is significantly larger than in any of the other colorings. As soon as these differences were noticed, we attempted to extend the coloring to 35 vertices and succeeded immediately. All 37 colorings found so far are closely related to $G_{1}$.

The coloring given in Table 1 is the most symmetric of the 37 colorings found to date. It has an automorphism group of order 4 [7]. There are six orbits of size 4 , consisting 
of vertices $i, i+1, i+2$ and $i+3$ for $i=0,4,8,12,16,20$. There are five orbits of size 2 , consisting of vertices $j$ and $j+1$ for $j=24,26,28,30,32$. Vertex 34 is a fixed point. Note that the minimum degree in color 1 is 11 and so the maximum degree in color 2 is 23 , which is one shy of the maximum possible, in view of the fact that $R(4,5)=25[10]$. Similarly, the minimum degree in color 2 is 18 , and so the maximum degree in color 1 is 16 , which is also one shy of the maximum possible, since $R(3,6)=18$ [10].

There are two other colorings that are adjacent to the coloring given above. They can be obtained from the original by recoloring the edge $1-3$, and by recoloring the edges $1-3$ and $29-33$. In both cases, the resulting coloring has an automorphism group of order two. In addition to these three colorings, another group of 34 good colorings was found nearby by reversing the colors of between 20 and 25 edges (it may be possible to obtain this latter group of colorings by a shorter route). This set of 34 colorings was originally found by a program that systematically looks for new colorings by changing the colors of small sets of edges. Later, some of the colorings in this set of 34 were also found by the Method B search program.

The Method B program that found these colorings has run to a successful conclusion (when searching for $(4,6)$-colorings on $K_{35}$ ) nearly 1000 times. In almost all of these cases, the coloring found was one of the three colorings that are mentioned at the begining of the previous paragraph. Only five times have we found a coloring in the group of 34 .

Copies of the 37 colorings, in different formats, can be found at the author's web site [4] and at Brendan McKay's web site [9].

\section{References}

[1] G. Exoo, A lower bound for R(5,5), J. Graph Theory 13 (1989) 97-98.

[2] G. Exoo, On two classical Ramsey numbers of the form $R(3, n)$, SIAM J. Discrete Math., 2 (1989) 488-490.

[3] G. Exoo, Announcement: On the Ramsey numbers $R(4,6), R(5,6)$, and $R(3,12)$, Ars Combin., 35 (1993) 85.

[4] G. Exoo, Ramsey numbers, http://ginger.indstate.edu/ge/RAMSEY/R46.

[5] F. Glover, Tabu search - Part 1, ORSA Journal on Computing 1 (1989) 190-206.

[6] S. Kirkpatrick, C. D. Gelatt and M. P. Vecchi, Optimization by simulated annealing, Science 220 (1983) 671-680.

[7] B. D. McKay, nauty User's Guide, Technical Report TR-CS-90-02, Dept. Comp. Sci., Australian National University (1990). Graph isomorphism/automorphism software, version 2.4 (2010), available at http://cs.anu . edu . au/ ^bdm/nauty.

[8] B. D. McKay and S. P. Radziszowski, Subgraph counting identities and Ramsey numbers, J. Combin. Th. Ser. B 69 (1997) 193-209.

[9] B. D. McKay, Ramsey graphs, http://cs.anu.edu.au/ bdm/data/ramsey.html.

[10] S. P. Radziszowski, Small Ramsey numbers, Electron. J. Combin., DS1, 2011. http://www . combinatorics.org/issue/view/Surveys. 